\title{
Micropropagação, aclimatização, teor e composição química do óleo essencial de genótipos de hortelã japonesa ${ }^{1}$
}

\author{
Micropropagation, acclimatization, essential oil content and chemical composition of \\ japanese mint genotypes
}

\author{
Maria de Fátima Arrigoni-Blank ${ }^{2 *}$, Andréa Santos Costa ${ }^{3}$, Valéria Oliveira Fonseca ${ }^{3}$, Pericles Barreto Alves ${ }^{4}$ e \\ Arie Fitzgerald Blank ${ }^{3}$
}

\begin{abstract}
Resumo - A hortelã japonesa (Mentha arvensis) é uma espécie aromática, originária do sul da China, o seu óleo essencial é rico em mentol, o qual é empregado nas indústrias farmacêuticas, de alimentos e de cosméticos. Este trabalho teve como objetivo desenvolver um protocolo para a micropropagação e aclimatização de genótipos de hortelã japonesa, além de analisar a composição química dos óleos essenciais de plantas micropropagadas e não micropropagadas. Segmentos nodais são os mais indicados para a micropropagação dos genótipos de $M$. arvensis. A concentração de 4,4 $\mu \mathrm{M}$ de AIA promoveu um maior número de brotos e folhas por explante do genótipo MA701-02, em relação aos demais genótipos. A utilização de 4,4 $\mu \mathrm{M}$ AIA e 9,3 $\mu \mathrm{M} \mathrm{CIN}+8,9 \mu \mathrm{M}$ BAP $+2,2 \mu \mathrm{M}$ AIA proporcionou maior acúmulo de massa seca no genótipo MA701-02, e no MA701-04 foi a concentração de 8,9 $\mu \mathrm{M}$ BAP $+5,4 \mu \mathrm{M}$ ANA. O substrato pó de coco $+1 \mathrm{~g} \mathrm{~L}^{-1}$ de calcário $+12 \mathrm{~g} \mathrm{~L}^{-1}$ de Biosafra ${ }^{\circledR}$ (3-12-6) pode ser indicado para a aclimatização de mudas micropropagadas dos genótipos de hortelã japonesa avaliados. A micropropagação não alterou o teor de óleo essencial e foram identificados 17 constituintes químicos, perfazendo um total de 92 a $99 \%$.
\end{abstract}

Palavras-chave - Mentha arvensis. Substrato. Planta medicinal e aromática. Cultura de tecidos. Mentol.

\begin{abstract}
Japanese mint (Mentha arvensis L.) is an aromatic species originated from South China and its essential oil is rich in menthol, which is used in the pharmaceutical, food and cosmetic industries. The aim of this work was to develop a protocol for micropropagation and acclimatization of japanese mint genotypes and analyze the chemical composition of the essential oils from micropropagated and not micropropagated plants. Nodal segments are the most indicated for micropropagation of the $M$. arvensis genotypes. The concentration of $4.4 \mu \mathrm{M}$ of IAA promoved higher number of shoots and leaves per explant of the MA701-02 genotype when compared with the others. The use of 4,4 $\mu \mathrm{M}$ of IAA and 9,3 $\mu \mathrm{M}$ of $\mathrm{KIN}+8,9 \mu \mathrm{M}$ of $\mathrm{BAP}+2,2 \mu \mathrm{M}$ of IAA resulted in major dry mass accumulation at genotype MA701-02. Major dry mass accumulation of genotype MA701-04 was promoted when 8,9 $\mu \mathrm{M}$ BAP $+5,4 \mu \mathrm{M}$ NAA was used. The substrate coconut dust $+1 \mathrm{~g} \mathrm{~L}^{-1}$ of limestone $+12 \mathrm{~g} \mathrm{~L}^{-1}$ of Biosafra ${ }^{\circledR}$ (3-12-6) can be indicated to acclimatization of micropropagated plantlets of the tested japanese mint genotypes. Micropropagation did not change essential oil content and 17 chemical constituents were indentified, totalizing 92 to $99 \%$.
\end{abstract}

Key words - Mentha arvensis. Substrate. Medicinal and aromatic plant. Tissue culture. Menthol.

\footnotetext{
* Autor para correspondência

${ }^{1}$ Recebido para publicação em 05/04/2010; aprovado em 03/02/2011

Pesquisa financiada pelo Edital CNPq 014/2004 - Fomento Tecnológico

${ }^{2}$ Departamento de Engenharia Agronomica, Universidade Federal de Sergipe, Av. Marechal Rondon s/n, São Cristóvão-SE, Brasil, 49.100-000, arrigoni@ufs.br

${ }_{3}^{3}$ Departamento de Engenharia Agronomica, Universidade Federal de Sergipe, São Cristóvão-SE, Brasil, deaasc@yahoo.com.br, valeriaoff81@ hotmail.com, afblank@ufs.br

${ }^{4}$ Departamento de Química, Universidade Federal de Sergipe, São Cristóvão-SE, Brasil, 49.100-000, pericles@ufs.br
} 


\section{Introdução}

A hortelã-japonesa (Mentha arvensis L.) é uma planta aromática, pertence à família Lamiaceae, conhecida também como hortelã pimenta, menta japonesa, hortelã, hortelã-doce, pimenta japonesa e vick. O seu óleo essencial se distingue de outras mentas pela ausência de 1,8 cineol e por elevado teor de mentol, empregado como flavorizante e aromatizante de alimentos, bebidas, perfumes, produtos de higiene bucal e preparações farmacêuticas, no tratamento de problemas respiratórios e gastrintestinais (KUMAR et al., 2002).

A micropropagação é uma técnica efetiva para multiplicação rápida de espécies nas quais é necessário obter alta uniformidade de progênie. Então, há um grande interesse de utilização destas técnicas para ampliar a propagação de plantas medicinais e aromáticas. $\mathrm{Na}$ propagação in vitro a composição e a concentração dos reguladores de crescimento no meio de cultura são fatores determinantes para o crescimento e padrão de desenvolvimento da maioria dos sistemas de cultivo in vitro (COSTA, 2006).

O sucesso na multiplicação in vitro do gênero Mentha tem sido estudado por diversos autores, os quais têm observado diferenças entre genótipos, explantes e reguladores de crescimento na micropropagação desta espécie. Explantes internodais de M. arvensis cultivares Himalaia e Kalka, quando inoculados em meio MS (MURASHIGE; SKOOG, 1962), contendo de 0,18 a $0,37 \mu \mathrm{M}$ de ácido naftaleno acético (ANA) e 1,12 a 2,25 $\mu \mathrm{M}$ de 6-benzilaminopurina (BAP), induziram a formação de calos, primórdios foliares e raízes (SHASANY et al., 1998). A utilização de segmentos nodais, em meio MS, suplementado com $0,09 \mu \mathrm{M}$ de ANA e $0,22 \mu \mathrm{M}$ de BAP, obtiveram 35 brotações por explante (ALI et al., 2004).

Um fator importante envolvido na aclimatização é o substrato utilizado na preparação das mudas, pois ele pode facilitar ou impedir o crescimento e desenvolvimento das plantas micropropagadas, conforme suas propriedades físico-químicas, influenciando diretamente no sucesso da aclimatização (COUTO et al., 2003, SHREBSKY et al., 2006).

O pó de coco representa uma das alternativas no preparo de substratos para a formação de mudas. Atualmente, o pó de coco tem sido indicado como substrato agrícola, principalmente, por apresentar uma estrutura física vantajosa, proporcionando alta porosidade, alto potencial de retenção de umidade e por ser biodegradável. É um meio de cultivo 100\% natural e indicado para germinação de sementes e propagação de plantas (ROSA et al., 2001).
O pó de coco é um subproduto considerado substrato praticamente inerte, que não reage com os nutrientes da adubação e possui longa durabilidade, sem alteração de suas características físicas. Como não possui os nutrientes essenciais para as plantas, deve ser utilizado em combinação com adubos (CARRIJO et al., 2002).

Este trabalho teve como objetivo desenvolver um protocolo para a micropropagação e aclimatização de genótipos de hortelã japonesa, além de analisar a composição química dos óleos essenciais de plantas micropropagadas e não micropropagadas.

\section{Material e métodos}

\section{Micropropagação}

O ensaio foi conduzido no Laboratório de Cultura de Tecidos Vegetais e Melhoramento Vegetal do Departamento de Engenharia Agronômica da Universidade Federal de Sergipe.

Como fontes de explantes foram utilizados segmento nodal, foliar e internodal proveniente de quatro genótipos de $M$. arvensis (UFC, MA701-01, MA701-02 e MA701-04) estabelecidos in vitro. O genótipo UFC foi doado pela Universidade Federal de Ceará e os demais genótipos foram doados pelo Instituto Agronômico de Campinas (IAC). O meio de cultivo utilizado foi o MS (MURASHIGE; SKOOG, 1962) acrescido de $30 \mathrm{~g} \mathrm{~L}^{-1}$ de sacarose e $7 \mathrm{~g} \mathrm{~L}^{-1}$ de ágar. $\mathrm{O} \mathrm{pH}$ do meio foi ajustado para $5,7 \pm 0,1 \mathrm{e}$, posteriormente, autoclavado $\left(121^{\circ} \mathrm{C} \mathrm{e}\right.$ $1,05 \mathrm{~atm})$ por 20 minutos. Após a inoculação, os frascos foram mantidos em sala de crescimento com fotoperíodo de 12 horas de luz, temperatura de $25 \pm 2{ }^{\circ} \mathrm{C}$ e intensidade luminosa de $60 \mu \mathrm{mol} \mathrm{m} \mathrm{m}^{-2} \mathrm{~s}^{-1}$. O delineamento experimental foi o inteiramente casualizado, com quatro tratamentos: meio MS (controle); MS suplementado com cinetina $(9,3 \mu \mathrm{M}), \mathrm{BAP}(8,9 \mu \mathrm{M})$ e ácido indolil acético (AIA) $(2,2 \mu \mathrm{M})$; MS suplementado de BAP $(8,9 \mu \mathrm{M})$ e ANA $(5,4 \mu \mathrm{M})$ e MS suplementado de AIA $(4,4 \mu \mathrm{M})$, sendo cada tratamento composto por seis repetições com quatro frascos, contendo dois explantes em cada frasco.

Aos 35 dias após implantação dos ensaios foram avaliadas as variáveis número de brotos, número de folhas e massa seca de parte área (MSPA). Os dados obtidos foram submetidos à análise de variância pelo teste $\mathrm{F}$ e, quando significativas, as médias foram comparadas pelo teste de Tukey $(\mathrm{p} \leq 0,05)$.

\section{Aclimatização}

O trabalho foi conduzido em estufa agrícola localizada no Departamento de Engenharia Agronômica 
na Universidade Federal de Sergipe. Foram utilizadas mudas micropropagadas dos genótipos UFC, MA70101, MA701-02, MA701-04 de M. arvensis. Após a lavagem em água corrente para eliminação do meio de cultura aderido às raízes, as mudas foram transplantadas para bandejas de poliestireno expandido com 72 células, contendo os diferentes substratos e mantidas sob irrigação e nebulização intermitentes e sombreamento de $50 \%$.

O delineamento experimental foi o inteiramente casualizado, em esquema fatorial $4 \mathrm{x} 4$, sendo quatro substratos: $\mathrm{PCB}$ - pó de coco $+1 \mathrm{~g} \mathrm{~L}^{-1}$ de calcário + $12 \mathrm{~g} \mathrm{~L}^{-1}$ de Biosafra ${ }^{\circledR}$ (3-12-6), PVCB (1:1 v/v) - pó de coco + vermiculita $+1 \mathrm{~g} \mathrm{~L}^{-1}$ de calcário $+12 \mathrm{~g} \mathrm{~L}^{-1}$ de Biosafra ${ }^{\circledR}(3-12-6)$, PVCB $(2: 1 \mathrm{v} / \mathrm{v})$ - pó de coco + vermiculita $+1 \mathrm{~g} \mathrm{~L}^{-1}$ de calcário $+12 \mathrm{~g} \mathrm{~L}^{-1}$ de Biosafra ${ }^{\circledR}$ (3-12-6) e VMS - vermiculita + sais do MS e quatro genótipos (UFC, MA701-01, MA701-02, MA701-04) com cinco repetições. Cada unidade experimental foi constituída por quatro mudas.

Aos 30 dias, foram avaliadas as variáveis porcentagem de sobrevivência (\%), número de brotos, comprimento de raiz $(\mathrm{cm})$, comprimento da parte aérea $(\mathrm{cm})$, massa fresca $(\mathrm{mg})$ da parte aérea (MFPA) e raiz (MFR), massa seca (mg) da parte aérea (MSPA) e raiz (MSR). As variáveis MFPA, MFR, MSR foram transformadas em raiz de $(x+0,5)$ e a porcentagem de sobrevivência em arco seno da raiz quadrada de (X/100). Os dados foram submetidos à análise de variância pelo teste $\mathrm{F}$ e, quando significativas, as médias foram comparadas pelo teste de Tukey $(\mathrm{p} \leq 0,05)$.

\section{Extração, teor e análise química do óleo essencial}

Plantas propagadas pelos métodos convencionais de estaquia e micropropagadas cultivadas em campo foram coletadas para extração do óleo essencial.

Os óleos essenciais de folhas frescas foram obtidos por hidrodestilação, através de aparelho do tipo clevenger (GUENTHER, 1972), por aproximadamente 2 horas. Os teores foram estimados com base no peso da matéria seca $\left(\mathrm{mL} 100 \mathrm{~g}^{-1}\right)$ e obtidos utilizando-se quatro amostras de $200 \mathrm{~g}$ (ASTA, 1968).

Amostras dos óleos essenciais foram analisadas por meio de cromatografia gasosa em equipamento Shimadzu QP5050A, interfaceada com espectroscopia de massa (CG/ EM), dotada de coluna capilar DB-5 (30 m x 0,25 mm x $0,25 \mu \mathrm{m})$ nas seguintes condições: gás de arraste hélio (fluxo $1,0 \mathrm{~mL} \mathrm{~min}{ }^{-1}$ ); tipo de injeção split a $250{ }^{\circ} \mathrm{C}(1 / 20)$; detector a $280{ }^{\circ} \mathrm{C}$, programação da coluna $80{ }^{\circ} \mathrm{C}$ durante 1,5 minuto, com aumento de $4{ }^{\circ} \mathrm{C}$ por minuto para $180{ }^{\circ} \mathrm{C}$, seguido de $10^{\circ} \mathrm{C}$ por minuto até $300^{\circ} \mathrm{C}$, finalizando com 10 minutos de isoterma a $300^{\circ} \mathrm{C}$. Os espectros de massas foram obtidos por impacto eletrônico a $70 \mathrm{eV}$. A identificação dos constituintes foi determinada com base na comparação do índice de retenção (VAN DEN DOOL; KRATZ, 1963) relativo a uma série de n-alcanos homólogos, obtido pela co-injeção de amostras do óleo com uma mistura linear de hidrocarbonetos, bem como com o banco de dados NIST21 e NIST107 da biblioteca do CG/EM e publicação de espectro de massas (ADAMS, 2007).

\section{Resultados e discussão}

\section{Micropropagação}

Os diferentes explantes utilizados apresentaram diferenças significativas para todas as variáveis analisadas, sendo o segmento nodal o único que proporcionou regeneração de plantas. Resultados diferentes foram obtidos por Shasany et al. (1998), onde os segmentos internodais foram eficientes na regeneração de $M$. arvensis cultivares Himalaia e Kalka. Isto sugere que, mesmo dentro de uma mesma espécie, podem ocorrer respostas diferentes, dependendo do explante utilizado, do local de cultivo, cultivares entre outros. Para o número de brotos, o segmento nodal dos genótipos MA70102 e UFC não apresentaram diferenças significativas, exceto pela combinação de $8,9 \mu \mathrm{M}$ BAP $+5,4 \mu \mathrm{M}$ ANA do genótipo MA701-01,que ficouabaixodamédia(0,5 brotos porexplante), apresentada pelo genótipo UFC ( 1,87 brotos por explante). O maior número de brotações foi observado no tratamento com 4,4 $\mu \mathrm{M}$ AIA, tanto para o genótipo MA701-02 como para o MA701-04 (TAB. 1). Chishti et al. (2006) obtiveram uma melhor multiplicação de brotos de $M$. arvensis, utilizando o meio MS suplementado de $8,9 \mu \mathrm{M}$ de BAP e 4,4 $\mu \mathrm{M}$ de AIA. Em Mentha viridis, o maior número de brotos foi obtido com segmento nodal com a concentração de 0,67 $\mu \mathrm{M}$ de BAP (RAJA; AROCKIASAMY, 2008).

Para número de folhas, não houve diferença significativa entre os genótipos na ausência de regulador de crescimento. O genótipo MA-701-02 obteve maior média em relação aos demais com a utilização de 4,4 $\mu \mathrm{M}$ AIA. Comparando os diferentes genótipos dentro dos tratamentos, não houve diferença significativa do número de folhas para os genótipos UFC e MA701-04. Para o genótipo MA70101, o tratamento $8,9 \mu \mathrm{M}$ BAP $+5,4 \mu \mathrm{M}$ ANA proporciou menor número de folhas. Para os genótipos MA701-01, UFC e MA701-04, a concentração de 4,4 $\mu \mathrm{M}$ AIA induziu menor número de folhas (TAB. 2). Em Mentha viridis, a maior média para esta variável foi encontrada no meio suplementado com $0,22 \mu \mathrm{M}$ BAP e zero de ANA, não diferindo estatisticamente dos meios contendo zero e $0,45 \mu \mathrm{M}$ BAP, combinados com 0,04 e 0,09 $\mu$ M ANA(LIMA etal., 2007). Na micropropagação de amoreira preta (Rubus spp.), o maior número de folhas foi encontrado com 0,11 $\mu \mathrm{M}$ BAP (VILLA et al., 2006). 
Tabela 1 - Número de brotos formados em explantes de M. arvensis, genótipos UFC, MA701-01, MA701-02, MA701-04, cultivados na presença de diferentes reguladores de crescimento

\begin{tabular}{|c|c|c|c|c|}
\hline \multirow[t]{3}{*}{ Tratamento $(\mu \mathrm{M})$} & \multicolumn{4}{|c|}{ Genótipos } \\
\hline & MA701-01 & MA701-02 & UFC & MA701-04 \\
\hline & \multicolumn{4}{|c|}{ Segmento foliar } \\
\hline Controle & $0,000 \mathrm{aA} \beta$ & $0,000 \mathrm{aA} \beta$ & $0,000 \mathrm{aA} \beta$ & $0,000 \mathrm{aA} \beta$ \\
\hline $9,3 \mathrm{CIN}+8,9 \mathrm{BAP}+2,2 \mathrm{AIA}$ & $0,000 \mathrm{aA} \beta$ & $0,000 \mathrm{aA} \beta$ & $0,000 \mathrm{aA} \beta$ & $0,000 \mathrm{aA} \beta$ \\
\hline $8,9 \mathrm{BAP}+5,4 \mathrm{ANA}$ & $0,000 \mathrm{aA} \beta$ & $0,000 \mathrm{aA} \beta$ & $0,000 \mathrm{aA} \beta$ & $0,000 \mathrm{aA} \beta$ \\
\hline \multirow[t]{2}{*}{ 4,4AIA } & $0,000 \mathrm{aA} \beta$ & $0,000 \mathrm{aA} \beta$ & $0,000 \mathrm{aA} \beta$ & $0,000 \mathrm{aA} \beta$ \\
\hline & \multicolumn{4}{|c|}{ Segmento nodal } \\
\hline Controle & $1,569 \mathrm{aA} \alpha$ & $1,694 \mathrm{bA} \alpha$ & $1,916 \mathrm{aA} \alpha$ & $2,027 \mathrm{bA} \alpha$ \\
\hline $9,3 \mathrm{CIN}+8,9 \mathrm{BAP}+2,2 \mathrm{AIA}$ & $1,708 \mathrm{aA} \alpha$ & $1,930 \mathrm{bA} \alpha$ & $1,875 \mathrm{aA} \alpha$ & $1,805 \mathrm{bA} \alpha$ \\
\hline $8,9 \mathrm{BAP}+5,4 \mathrm{ANA}$ & $0,500 \mathrm{bB} \alpha$ & $1,916 \mathrm{bA} \alpha$ & $1,875 \mathrm{aA} \alpha$ & $0,666 \mathrm{cB} \alpha$ \\
\hline \multirow[t]{2}{*}{ 4,4AIA } & $1,500 \mathrm{aC} \alpha$ & 4,408 aA $\alpha$ & $2,000 \mathrm{aBC} \alpha$ & $2,569 \mathrm{aB} \alpha$ \\
\hline & \multicolumn{4}{|c|}{ Segmento internodal } \\
\hline Controle & $0,000 \mathrm{aA} \beta$ & $0,000 \mathrm{aA} \beta$ & $0,000 \mathrm{aA} \beta$ & $0,000 \mathrm{aA} \beta$ \\
\hline $9,3 \mathrm{CIN}+8,9 \mathrm{BAP}+2,2 \mathrm{AIA}$ & $0,000 \mathrm{aA} \beta$ & $0,000 \mathrm{aA} \beta$ & $0,000 \mathrm{aA} \beta$ & $0,000 \mathrm{aA} \beta$ \\
\hline $8,9 \mathrm{BAP}+5,4 \mathrm{ANA}$ & $0,000 \mathrm{aA} \beta$ & $0,000 \mathrm{aA} \beta$ & $0,000 \mathrm{aA} \beta$ & $0,000 \mathrm{aA} \beta$ \\
\hline 4,4AIA & $0,000 \mathrm{aA} \beta$ & $0,000 \mathrm{aA} \beta$ & $0,000 \mathrm{aA} \beta$ & $0,000 \mathrm{aA} \beta$ \\
\hline $\mathrm{CV}(\%)$ & \multicolumn{4}{|c|}{14,73} \\
\hline
\end{tabular}

* Médias seguidas das mesmas letras minúsculas nas colunas, maiúsculas nas linhas e gregas entre segmentos, não diferem estatisticamente entre si, pelo teste de Tukey $(\mathrm{p} \leq 0,05)$

Tabela 2 - Número de folhas por explante de M. arvensis, genótipos UFC, MA701-01, MA701-02 e MA701-04, cultivados na presença de diferentes reguladores de crescimento

\begin{tabular}{|c|c|c|c|c|}
\hline \multirow[t]{3}{*}{ Tratamento $(\mu \mathrm{M})$} & \multicolumn{4}{|c|}{ Genótipos } \\
\hline & MA701-01 & MA701-02 & UFC & MA701-04 \\
\hline & \multicolumn{4}{|c|}{ Segmento Foliar } \\
\hline Controle & $0,000 \mathrm{aA} \beta$ & $0,000 \mathrm{aA} \beta$ & $0,000 \mathrm{aA} \beta$ & $0,000 \mathrm{aA} \beta$ \\
\hline $9,3 \mathrm{CIN}+8,9 \mathrm{BAP}+, 2 \mathrm{AIA}$ & $0,000 \mathrm{aA} \beta$ & 0,000 aA $\beta$ & $0,000 \mathrm{aA} \beta$ & $0,000 \mathrm{aA} \beta$ \\
\hline $8,9 \mathrm{BAP}+5,4 \mathrm{ANA}$ & $0,000 \mathrm{aA} \beta$ & $0,000 \mathrm{aA} \beta$ & $0,000 \mathrm{aA} \beta$ & $0,000 \mathrm{aA} \beta$ \\
\hline \multirow[t]{2}{*}{ 4,4AIA } & $0,000 \mathrm{aA} \beta$ & $0,000 \mathrm{aA} \beta$ & $0,000 \mathrm{aA} \beta$ & $0,000 \mathrm{aA} \beta$ \\
\hline & \multicolumn{4}{|c|}{ Segmento Nodal } \\
\hline Controle & $14,472 \mathrm{aA} \alpha$ & $17,028 \mathrm{bA} \alpha$ & $12,833 \mathrm{aA} \alpha$ & $12,930 \mathrm{aA} \alpha$ \\
\hline 9,3CIN+8,9BAP+2,2AIA & $12,583 \mathrm{aAB} \alpha$ & $9,778 \mathrm{cAB} \alpha$ & $14,083 \mathrm{aA} \alpha$ & $9,611 \mathrm{aB} \alpha$ \\
\hline $8,9 \mathrm{BAP}+5,4 \mathrm{ANA}$ & $1,833 \mathrm{bB} \alpha$ & $11,638 \mathrm{cA} \alpha$ & $14,291 \mathrm{aA} \alpha$ & $12,500 \mathrm{aA} \alpha$ \\
\hline \multirow[t]{2}{*}{ 4,4AIA } & $14,333 \mathrm{aB} \alpha$ & $23,042 \mathrm{aA} \alpha$ & $16,542 \mathrm{aB} \alpha$ & $13,388 \mathrm{aB} \alpha$ \\
\hline & \multicolumn{4}{|c|}{ Segmento Internodal } \\
\hline Controle & $0,000 \mathrm{bA} \beta$ & $0,000 \mathrm{aA} \beta$ & $0,000 \mathrm{aA} \beta$ & 0,000 aA $\beta$ \\
\hline $9,3 \mathrm{CIN}+8,9 \mathrm{BAP}+2,2 \mathrm{AIA}$ & $0,000 \mathrm{aB} \beta$ & $0,000 \mathrm{aA} \beta$ & $0,000 \mathrm{aA} \beta$ & $0,000 \mathrm{aA} \beta$ \\
\hline $8,9 \mathrm{BAP}+5,4 \mathrm{ANA}$ & $0,000 \mathrm{bA} \beta$ & $0,000 \mathrm{aA} \beta$ & 0,000 aA $\beta$ & 0,000 aA $\beta$ \\
\hline 4,4AIA & $0,000 \mathrm{bA} \beta$ & $0,000 \mathrm{aA} \beta$ & $0,000 \mathrm{aA} \beta$ & $0,000 \mathrm{aA} \beta$ \\
\hline $\mathrm{CV}(\%)$ & 25,72 & & & \\
\hline
\end{tabular}

* Médias seguidas das mesmas letras minúsculas nas colunas, maiúsculas nas linhas e gregas entre segmentos, não diferem estatisticamente entre si, pelo teste de Tukey $(\mathrm{p} \leq 0,05)$ 
O uso de citocininas estimula maior produção de parte aérea, através do aumento da massa fresca, número de gemas e folhas, porém o contrário é obtido com a utilização de concentrações mais elevadas, acima da ótima para a multiplicação da espécie em estudo (GRATTAPAGLIA; MACHADO, 1998).

Em relação à MSPA, houve diferença significativa entre os genótipos, sendo a utilização de 4,4 $\mu \mathrm{M}$ AIA e 9,3 $\mu \mathrm{M}$ CIN $+8,9 \mu \mathrm{M}$ BAP $+2,2 \mu \mathrm{M}$ AIA as que proporcionaram o maior acúmulo de massa seca no genótipo MA701-02 . Já para o genótipo MA701-04, o tratamento com 9,3 $\mu \mathrm{M}$ CIN $+8,9 \mu \mathrm{M}$ BAP $+2,2 \mu \mathrm{M}$ AIA proporcionou um menor acúmulo de massa. Para o genótipo MA701-01, as concentrações de 8,9 $\mu \mathrm{M}$ BAP + $5,4 \mu \mathrm{M}$ ANA fizeram com que esse obtivesse uma massa seca de parte aérea insignificante, porém não havendo diferença significativa entre os demais tratamentos avaliados, os quais proporcionaram maior acúmulo de massa. O genótipo UFC não apresentou diferença significativa entre os tratamentos avaliados, entretanto, quando comparado aos genótipos, apresentou diferença significativa, onde este obteve os menores acúmulos de massa, com exceção no tratamento $8,9 \mu \mathrm{M}$ BAP $+5,4 \mu \mathrm{M}$ ANA, que foi igual ao MA701-01 (TAB. 3). Em manjericão (Ocimum basilicum L.), os maiores acúmulos de massa seca da parte aérea $(0,047 ; 0,046$ e $0,042 \mathrm{~g})$ foram obtidos com 0,$04 ; 0,09$ e $0,18 \mu \mathrm{M}$ de ANA, respectivamente (SOUZA et al., 2006).

\section{Aclimatização}

$\mathrm{Na}$ implantação do ensaio, as mudas micropropagadas apresentavam altura média de $6,0 \mathrm{~cm}$ e resultaram em altas taxas de sobrevivência para todos os substratos testados, havendo diferença significativa entre eles (TAB. 4).

Para a variável número de brotos, não houve diferença significativa dos genótipos entre os diferentes substratos. Entretanto, para os genótipos nos substratos, observa-se que o UFC teve as maiores médias, não diferindo estatisticamente do genótipo MA701-01, em todos os substratos, e do MA701-02, nos substratos PVCB $(1: 1 \mathrm{v} / \mathrm{v})$ e VMS.

Já para comprimento de raiz, nota-se que não houve diferença significativa entre os substratos avaliados para os genótipos MA701-01 e MA701-04, enquanto que, para UFC e MA701-02, houve diferença significativa entre os substratos testados. Comparando os genótipos, dentro do substrato PVCB (2:1 v/v), o genótipo MA701-01 obteve o menor comprimento de raiz e, no substrato PCB, os

Tabela 3 - Massa seca de parte aérea de M. arvensis, genótipos UFC, MA701-01, MA 701-02 e MA701-04, cultivados na presença de diferentes reguladores de crescimento

\begin{tabular}{lcccc}
\hline \multicolumn{1}{c}{ Tratamento $(\mu \mathrm{M})$} & \multicolumn{4}{c}{ Genótipos } \\
\hline & MA701-01 & MA701-02 & UFC & MA701-04 \\
\cline { 2 - 4 } Controle & $0,000 \mathrm{aA} \beta$ & $0,000 \mathrm{aA} \beta$ & $0,000 \mathrm{aA} \alpha$ & $0,000 \mathrm{aA} \beta$ \\
9,3CIN+8,9BAP+2,2AIA & $0,000 \mathrm{aA} \gamma$ & $0,000 \mathrm{aA} \beta$ & $0,001 \mathrm{aA} \alpha$ & $0,000 \mathrm{aA} \beta$ \\
8,9BAP+5,4ANA & $0,000 \mathrm{aA} \alpha$ & $0,000 \mathrm{aA} \beta$ & $0,000 \mathrm{aA} \alpha$ & $0,000 \mathrm{aA} \beta$ \\
4,4AIA & $0,000 \mathrm{aA} \beta$ & $0,000 \mathrm{aA} \beta$ & $0,000 \mathrm{aA} \alpha$ & $0,000 \mathrm{aA} \beta$ \\
\hline & & Segmento Nodal & $5,217 \mathrm{aA} \alpha$ \\
Controle & $5,447 \mathrm{aA} \alpha$ & $4,937 \mathrm{bA} \alpha$ & $0,008 \mathrm{aB} \alpha$ & $3,454 \mathrm{bC} \alpha$ \\
9,3CIN+8,9BAP+2,2AIA & $5,221 \mathrm{aB} \alpha$ & $8,307 \mathrm{aA} \alpha$ & $0,007 \mathrm{aD} \alpha$ & $4,321 \mathrm{abA} \alpha$ \\
8,9BAP+5,4ANA & $0,000 \mathrm{bC} \alpha$ & $2,989 \mathrm{cB} \alpha$ & $0,006 \mathrm{aC} \alpha$ & $3,814 \mathrm{abC} \alpha$ \\
4,4AIA & $6,579 \mathrm{aB} \alpha$ & $9,512 \mathrm{aA} \alpha$ & $0,007 \mathrm{aD} \alpha$ & $0,000 \mathrm{aA} \beta$ \\
\hline & & $\mathrm{Segmento}$ Internodal & $0,000 \mathrm{aB} \beta$ \\
Controle & $0,000 \mathrm{bA} \beta$ & $0,000 \mathrm{aA} \beta$ & $0,000 \mathrm{aA} \alpha$ \\
9,3CIN+8,9BAP+2,2AIA & $0,000 \mathrm{aA} \beta$ & $0,000 \mathrm{aB} \beta$ & $0,000 \mathrm{aB} \alpha$ & $0,000 \mathrm{aA} \beta$ \\
4,9BAP+5,4ANA & $0,000 \mathrm{bA} \alpha$ & $0,000 \mathrm{aA} \beta$ & $0,000 \mathrm{aA} \alpha$ & $0,000 \mathrm{aA} \beta$ \\
\hline \multicolumn{1}{c}{ CV(\%) } & $0,000 \mathrm{bA} \beta$ & $0,000 \mathrm{aA} \beta$ & $0,000 \mathrm{aA} \alpha$ & \\
\hline
\end{tabular}

* Médias seguidas das mesmas letras minúsculas nas colunas, maiúsculas nas linhas e gregas entre segmentos, não diferem estatisticamente entre si, pelo teste de Tukey $(\mathrm{p} \leq 0,05)$ 
maiores comprimentos de raízes foram obtidos com os genótipos UFC, MA701-01 e MA701-04 (TAB. 4). O pó de coco proprocionou uma maior agregação das raízes ao substrato, facilitando a retirada da muda da bandeja e o desenvolvimento da mesma. Isso pode estar relacionado ao fato do pó de coco seco possuir características físicas e químicas adequadas para o crescimento e desenvolvimento desses genótipos. Para a produção de mudas de tomate, o pó de coco puro não se revelou bom substrato, pois nele as plântulas não apresentaram bom desenvolvimento (SILVEIRA et al., 2002).

O substrato VMS proporcionou um maior comprimento da parte aérea para o MA701-01 (TAB. 4). No entanto, para aclimatização de mudas de abacaxi, a vermiculita apresentou baixa agregação com as raízes, o que afetou negativamente o crescimento das plantas, tanto da parte aérea quanto das raízes (MOREIRA et al., 2006).

Tabela 4 - Valores médios de porcentagem de sobrevivência (\%), número de brotos, comprimento de raiz, comprimento de parte aérea, massa fresca e seca de parte aérea e raiz por planta, em função de diferentes substratos na aclimatização dos genótipos de hortelã japonesa

\begin{tabular}{|c|c|c|c|c|}
\hline \multirow[t]{2}{*}{ Substrato } & \multicolumn{4}{|c|}{ Genótipos } \\
\hline & UFC & MA701-01 & MA701-02 & MA701-04 \\
\hline & \multicolumn{4}{|c|}{ Porcentagem de Sobrevivência (\%) } \\
\hline PCB & 100,00 a $\mathrm{A}$ & 95,00 a $\mathrm{AB}$ & $90,00 \mathrm{~b} \mathrm{~B}$ & 100,00 a $\mathrm{A}$ \\
\hline $\operatorname{PVCB}(1: 1 \mathrm{v} / \mathrm{v})$ & 100,00 a A & 95,00 a A & 100,00 a A & 100,00 a $\mathrm{A}$ \\
\hline $\operatorname{PVCB}(2: 1 \mathrm{v} / \mathrm{v})$ & 100,00 a A & 95,00 a $\mathrm{A}$ & 100,00 a A & 100,00 a A \\
\hline VMS & 100,00 a A & 100,00 a A & 100,00 a A & 100,00 a A \\
\hline \multirow[t]{2}{*}{$\mathrm{CV}(\%)$} & \multicolumn{4}{|c|}{5,29} \\
\hline & \multicolumn{4}{|c|}{ Número de brotos } \\
\hline PCB & 8,90 a $\mathrm{A}$ & 6,35 a $\mathrm{AB}$ & 4,80 a B & 4,65 a B \\
\hline $\operatorname{PVCB}(1: 1 \mathrm{v} / \mathrm{v})$ & 10,00 a $\mathrm{A}$ & 7,10 a $\mathrm{AB}$ & 6,50 a $\mathrm{AB}$ & 5,75 a B \\
\hline $\operatorname{PVCB}(2: 1 \mathrm{v} / \mathrm{v})$ & 10,80 a A & 8,25 a $\mathrm{AB}$ & 6,70 a B & 5,55 a B \\
\hline VMS & 10,90 a A & 8,40 a $\mathrm{AB}$ & 8,25 a $\mathrm{AB}$ & 6,50 a B \\
\hline \multirow[t]{2}{*}{$\mathrm{CV}(\%)$} & \multicolumn{4}{|c|}{27,79} \\
\hline & \multicolumn{4}{|c|}{ Comprimento de raiz $(\mathrm{cm})$} \\
\hline PCB & 15,60 a $\mathrm{A}$ & 13,17 a $\mathrm{AB}$ & $10,96 \mathrm{c} \mathrm{B}$ & 13,75 a $\mathrm{AB}$ \\
\hline $\operatorname{PVCB}(1: 1 \mathrm{v} / \mathrm{v})$ & $14,87 \mathrm{ab} \mathrm{A}$ & 12,15 a A & $12,55 \mathrm{bc} \mathrm{A}$ & 14,51 a $\mathrm{A}$ \\
\hline $\operatorname{PVCB}(2: 1 \mathrm{v} / \mathrm{v})$ & $14,60 \mathrm{ab} \mathrm{A}$ & 10,77 a B & 17,37 a A & 15,43 a A \\
\hline VMS & $12,11 \mathrm{~b} \mathrm{~A}$ & 14,07 a A & $14,90 \mathrm{ab} \mathrm{A}$ & 13,85 a $\mathrm{A}$ \\
\hline \multirow[t]{2}{*}{$\mathrm{CV}(\%)$} & \multicolumn{4}{|c|}{13,05} \\
\hline & \multicolumn{4}{|c|}{ Comprimento de parte aérea $(\mathrm{cm})$} \\
\hline PCB & 23,05 a $\mathrm{A}$ & $11,20 \mathrm{~b} \mathrm{C}$ & $19,92 \mathrm{c} \mathrm{A}$ & $15,47 \mathrm{~b} \mathrm{~B}$ \\
\hline $\operatorname{PVCB}(1: 1 \mathrm{v} / \mathrm{v})$ & 25,90 a A & $10,75 \mathrm{~b} \mathrm{C}$ & $24,79 \mathrm{~b} \mathrm{~A}$ & $19,07 \mathrm{ab} \mathrm{B}$ \\
\hline $\operatorname{PVCB}(2: 1 \mathrm{v} / \mathrm{v})$ & 24,09 a $\mathrm{AB}$ & 9,45 b C & $26,03 \mathrm{ab} A$ & 21,49 a B \\
\hline VMS & 22,97 a B & 16,35 a C & 29,73 a A & 21,57 a B \\
\hline \multirow[t]{2}{*}{$\mathrm{CV}(\%)$} & \multicolumn{4}{|c|}{11,04} \\
\hline & \multicolumn{4}{|c|}{ Massa fresca de parte aérea $(\mathrm{mg})$} \\
\hline PCB & 4646,00 a $\mathrm{A}$ & $1172,00 \mathrm{ab} B$ & $1355,00 \mathrm{~b} \mathrm{~B}$ & $757,50 \mathrm{~b} \mathrm{~B}$ \\
\hline $\operatorname{PVCB}(1: 1 \mathrm{v} / \mathrm{v})$ & 4832,35 a A & $1220,35 \mathrm{ab} C$ & $2490,00 \mathrm{~b} \mathrm{~B}$ & $1159,50 \mathrm{ab} C$ \\
\hline $\operatorname{PVCB}(2: 1 \mathrm{v} / \mathrm{v})$ & 4252,10 a A & 868,95 b B & $2005,00 \mathrm{~b} \mathrm{C}$ & 2107,50 a B \\
\hline VMS & 2640,85 b B & 2325,55 a B & 4190,00 a A & $1790,00 \mathrm{ab} B$ \\
\hline $\mathrm{CV}(\%)$ & \multicolumn{4}{|c|}{13,40} \\
\hline
\end{tabular}


Continuação da Tabela 4

\begin{tabular}{|c|c|c|c|c|}
\hline \multirow[b]{2}{*}{ PCB } & \multicolumn{4}{|c|}{ Massa fresca de raiz (mg) } \\
\hline & 570,80 a B & 712,20 a $\mathrm{AB}$ & $85,00 \mathrm{c} \mathrm{C}$ & 1040,00 a A \\
\hline $\operatorname{PVCB}(1: 1 \mathrm{v} / \mathrm{v})$ & $414,35 \mathrm{ab} \mathrm{AB}$ & $524,05 \mathrm{ab} A$ & $185,00 \mathrm{bc} \mathrm{B}$ & 655,00 a $\mathrm{A}$ \\
\hline $\operatorname{PVCB}(2: 1 \mathrm{v} / \mathrm{v})$ & $192,00 \mathrm{bc} \mathrm{B}$ & 282,05 b B & 635,00 a $\mathrm{A}$ & 890,00 a $\mathrm{A}$ \\
\hline VMS & $62,10 \mathrm{c} \mathrm{C}$ & $303,10 \mathrm{~b} \mathrm{~B}$ & $375,00 \mathrm{ab} \mathrm{B}$ & 985,00 a $\mathrm{A}$ \\
\hline \multirow[t]{2}{*}{$\mathrm{CV}(\%)$} & \multicolumn{4}{|c|}{20,08} \\
\hline & \multicolumn{4}{|c|}{ Massa seca de parte aérea (mg) } \\
\hline PCB & 512,95 a $\mathrm{A}$ & $203,40 \mathrm{~b} \mathrm{~B}$ & $217,90 \mathrm{~b} \mathrm{~B}$ & 126,25 a B \\
\hline $\operatorname{PVCB}(1: 1 \mathrm{v} / \mathrm{v})$ & 633,00 a $\mathrm{A}$ & $236,65 \mathrm{~b}$ B & $289,25 \mathrm{~b} \mathrm{~B}$ & 174,35 a B \\
\hline $\operatorname{PVCB}(2: 1 \mathrm{v} / \mathrm{v})$ & 598,50 a A & 184,55 b B & 289,55 b B & 200,85 a B \\
\hline VMS & 513,75 a A & 508,75 a A & 546,80 a $\mathrm{A}$ & 313,30 a B \\
\hline \multirow[t]{2}{*}{$\mathrm{CV}(\%)$} & \multicolumn{4}{|c|}{29,90} \\
\hline & \multicolumn{4}{|c|}{ Massa seca de raiz (mg) } \\
\hline $\mathrm{PCB}$ & 55,50 a $\mathrm{AB}$ & $65,71 \mathrm{ab} \mathrm{A}$ & $28,60 \mathrm{c} \mathrm{B}$ & 65,00 a $\mathrm{A}$ \\
\hline $\operatorname{PVCB}(1: 1 \mathrm{v} / \mathrm{v})$ & 71,95 a $\mathrm{A}$ & $45,28 \mathrm{~b} \mathrm{AB}$ & $35,50 \mathrm{bc} \mathrm{B}$ & 52,40 a $\mathrm{AB}$ \\
\hline $\operatorname{PVCB}(2: 1 \mathrm{v} / \mathrm{v})$ & 76,35 a $\mathrm{A}$ & $56,83 \mathrm{ab} A$ & 87,50 a A & 79,45 a A \\
\hline VMS & 51,05 a A & 91,26 a $\mathrm{A}$ & $74,95 \mathrm{ab} \mathrm{A}$ & 74,75 a A \\
\hline $\mathrm{CV}(\%)$ & & & & \\
\hline
\end{tabular}

* Médias seguidas das mesmas letras minúsculas nas colunas e maiúsculas nas linhas, não diferem estatisticamente entre si pelo teste de Tukey $(\mathrm{p} \leq 0,05)$; Substratos: PCB - pó de coco +1 g.L.-1 de calcário +12 g.L.1 de Biosafra ${ }^{\circledR}(3-12-6)$, PVCB $(1: 1$ v/v) - pó de coco + vermiculita + 1 g.L. ${ }^{-1}$ de calcário +12 g. $L^{-1}$ de Biosafra ${ }^{\circledR}(3-12-6)$, PVCB $\left(2: 1\right.$ v/v) - pó de coco + vermiculita +1 g.L. ${ }^{-1}$ de calcário +12 g.L $L^{-1}$ de Biosafra ${ }^{\circledR}$ (3-12-6) e VMS - vermiculita + sais do MS

Em relação ainda a esta variável, o MA70104 apresentou menor comprimento de parte aérea em PCB e PVCB (1:1 v/v). Resultados semelhantes foram encontrados na produção de mudas de porta-enxertos de gravioleira, onde o substrato pó de coco + vermiculita $(1: 1 \mathrm{v} / \mathrm{v})$ promoveu menor altura das plantas (CORREIA et al.,2001). Nota-se que o MA701-01 apresentou as menores médias, em relação ao UFC, MA701-02 e ao MA701-04, quando avaliado para cada substrato com os demais genótipos (TAB. 4). Esse pequeno comprimento da parte aérea do genótipo MA701-01 pode ter ocorrido em virtude do mesmo ser adaptado às condições da região Sul e Sudeste (GRISI et al, 2006). Resultados contrários aos deste trabalho, em relação ao comprimento da parte aérea das plantas, foram encontrados na aclimatização de antúrio, onde o substrato pó de coco seco promoveu maior altura de plântula $(8,87 \mathrm{~cm})$ (SILVA et al., 2007). Oliveira et al. (2008) também observaram os efeitos benéficos deste substrato, visto que alcançaram melhor desenvolvimento vegetativo das cultivadas em pó de coco seco.

Para MFPA, o genótipo UFC apresentou menor biomassa, quando se usou substrato VMS, e para o genótipo MA701-02 este substrato proporcionou maior biomassa. Não houve diferença significativa entre os substratos PCB, PVCB (1:1 v/v) e PVCB $(2: 1 \mathrm{v} / \mathrm{v})$ para os genótipos UFC e MA701-02. Para o genótipo MA70101 o substrato VMS foi superior ao $\operatorname{PVCB}(2: 1 \mathrm{v} / \mathrm{v})$, não diferenciando dos substratos PCB e PVCB (1:1 v/v). Já para o genótipo MA701-04 o substrato PVCB (2:1 v/v) foi superior ao PCB, não diferenciando dos substratos PVCB $(1: 1 \mathrm{v} / \mathrm{v})$ e VMS. Comparando os genótipos dentro de cada substrato, percebe-se que o genótipo UFC teve maior acúmulo de MFPA, em relação aos demais genótipos nos substratos PCB, PVCB (1:1 v/v) e PVCB (2:1 v/v) e, no substrato VMS, foi o genótipo MA701-02 (TAB. 4). Na aclimatização de mudas de bromélia Aechmea nudicalis, os melhores resultados para comprimento da parte aérea, matéria fresca da parte aérea e das raízes e matéria seca das raízes foram observados em misturas contendo o pó de coco seco (ANDRADA-FRANCO; RODRIGUEZ, 2003).

Os genótipos UFC e MA701-01 obtiveram as maiores MFR, nos substratos PCB e PVCB (1:1 v/v), e o MA701-02 em PVCB (2:1 v/v) e VMS e, para o genótipo MA701-04, não houve diferença significativa entre os substratos testados. Avaliando os genótipos dentro de cada substrato, percebe-se que a maior MFR foi obtida pelo genótipo MA701-04, no 
substrato VMS, e esse genótipo foi igual ao MA701-02, no substrato PVCB $(2: 1 \mathrm{v} / \mathrm{v})$. No substrato PCB, o genótipo obteve a MA701-02 menor MFR.

Para a variável MSPA, o MA701-01 e o MA701-02 expressaram as maiores massa seca no substrato VMS, ao contrário do MA701-04 e do UFC, que não apresentaram diferenças significativas entre os substratos testados. $\mathrm{O}$ genótipo UFC apresentou os maiores valores, se destacando dos demais, em relação aos substratos avaliados, porém não deferindo dos genótipos MA701-01 e MA701-02, no substrato VMS (TAB. 4). O substrato pó de coco seco propiciou maior peso de matéria seca da parte aérea $(0,18 \mathrm{~g})$, na aclimatização de antúrio (SILVA et al., 2007).

Verificou-se que o substrato VMS expressou maior biomassa para o MA701-01, para a MSR, não deferindo estatisticamente do PCB e PVCB $(2: 1 \mathrm{v} / \mathrm{v})$. Para o genótipo MA701-02, o substrato PVCB (2:1 v/v) propiciou uma maior MSR, porém não deferindo do VMS. Esse último substrato também proporcionou maior MSR, em plantas de antúrio aclimatizadas (SILVA et al., 2007). Para os genótipos testados, houve diferença significativa entre eles, para os substratos PCB e PVCB (1:1 v/v), sendo o genótipo MA701-02 o que apresentou menor massa, porém não deferiu dos genótipos UFC, MA70101, MA701-04, respectivamente (TAB. 4).

Com relação à aclimatização da espécie $M$. arvensis, os resultados obtidos, na Tabela 4, demonstram que o pó de coco constitui em material promissor para ser utilizado na aclimatização das mudas dos genótipos avaliados, uma vez que este substrato proporcionou condições favoráveis para o melhor desenvolvimento das mudas e também por ser um subproduto abundante da agroindústria do coco, de ampla disponibilidade no Nordeste do Brasil e de baixo valor no mercado, barateando o custo de produção.

\section{Análise do óleo essencial}

Dos componentes presentes no óleo essencial dos quatro genótipos de $M$. arvensis, propagadas

Tabela 5 - Composição química do óleo essencial de genótipos de M. arvensis, submetidos à propagação convencional e micropropagação

\begin{tabular}{|c|c|c|c|c|c|c|c|c|c|}
\hline \multirow{3}{*}{ Composto $^{\mathrm{a}}$} & \multirow{3}{*}{$\mathrm{IR}^{\mathrm{b}}$} & \multicolumn{8}{|c|}{ Genótipos } \\
\hline & & \multicolumn{2}{|c|}{ UFC } & \multicolumn{2}{|c|}{ MA701-01 } & \multicolumn{2}{|c|}{ MA701-02 } & \multicolumn{2}{|c|}{ MA701-04 } \\
\hline & & Conv. & Micro & Conv. & Micro & Conv. & Micro & Conv. & Micro \\
\hline & & ............. & ........ & $\ldots \ldots \ldots$ & ............... & ............. & ................ & ................. & $\ldots \ldots \ldots$ \\
\hline Tricicleno & 929 & 0,08 & 0,84 & 0,00 & 0,00 & 0,42 & 0,00 & 0,17 & 0,00 \\
\hline$\beta$-Pineno & 973 & 0,18 & 1,18 & 0,00 & 0,68 & 0,77 & 0,53 & 0,23 & 0,30 \\
\hline Mirceno & 985 & 0,08 & 0,00 & 0,00 & 0,00 & 0,21 & 1,39 & 0,00 & 0,84 \\
\hline 3-Octanol & 992 & 0,48 & 0,00 & 0,00 & 0,00 & 0,27 & 0,00 & 0,18 & 4,21 \\
\hline Limoneno & 1025 & 1,22 & 10,65 & 0,00 & 3,85 & 0,54 & 0,31 & 1,42 & 0,00 \\
\hline 1,8-Cineol & 1029 & 0,00 & 0,00 & 0,00 & 0,00 & 12,16 & 9,94 & 0,00 & 0,00 \\
\hline Linalol & 1096 & 0,19 & 0,00 & 0,00 & 0,00 & 0,00 & 0,00 & 0,00 & 0,00 \\
\hline Mentona & 1153 & 14,29 & 11,93 & 14,66 & 25,94 & 20,46 & 12,69 & 0,00 & 0,00 \\
\hline Isomentona & 1161 & 1,81 & 2,03 & 2,87 & 6,50 & 6,04 & 3,06 & 0,37 & 0,00 \\
\hline Neomentol & 1166 & 1,51 & 0,90 & 0,00 & 0,00 & 0,00 & 0,00 & 0,00 & 0,00 \\
\hline Isopulegona & 1172 & 0,07 & 0,00 & 0,00 & 0,00 & 0,00 & 0,00 & 2,53 & 0,00 \\
\hline Mentol & 1173 & 77,85 & 69,07 & 81,10 & 53,26 & 0,00 & 0,00 & 0,00 & 0,00 \\
\hline$\alpha$-Terpineol & 1192 & 0,21 & 0,00 & 0,00 & 0,00 & 1,09 & 0,00 & 0,00 & 0,00 \\
\hline Pulegona & 1234 & 0,26 & 0,00 & 0,00 & 0,75 & 51,56 & 68,25 & 48,04 & 55,55 \\
\hline Piperitona & 1249 & 1,17 & 0,00 & 0,00 & 1,56 & 0,29 & 0,00 & 0,00 & 0,00 \\
\hline Acetato de mentila & 1286 & 0,27 & 2,98 & 1,35 & 1,79 & 0,00 & 0,00 & 0,00 & 0,00 \\
\hline Piperitenona & 1338 & 0,00 & 0,00 & 0,00 & 0,00 & 0,00 & 0,00 & 40,25 & 31,85 \\
\hline $\begin{array}{l}\text { Teor de óleo essencial em } \\
\text { folha seca }(\%)\end{array}$ & & 3,16 & 3,10 & 4,59 & 4,67 & 1,60 & 1,66 & 1,90 & 1,98 \\
\hline Total Identificado & & 99,67 & 99,57 & 99,98 & 95,33 & 93,81 & 96,17 & 93,19 & 92,75 \\
\hline
\end{tabular}

${ }^{a}$ Compostos listados em ordem de eluição em coluna DB-5; ${ }^{b}$ Indice de Retenção; Conv.: propagação convencional; Micro: micropropagação 
convencionalmente por estaquia e micropropagadas, 17 constituintes foram identificados e encontram-se listados de acordo com a ordem de eluição (TAB. 5).

Analisando o genótipo UFC, observa-se que os principais compostos obtidos pelos métodos convencionais de propagação por estaquia e propagação in vitro foram, respectivamente, o mentol, com $77,85 \%$ e $69,07 \%$, e a mentona, com $14,29 \%$ e $11,93 \%$. No genótipo MA701-01, houve uma redução do teor de mentol em plantas micropropagadas de 81,1 para $53,26 \%$ e um aumento no teor de mentona de 14,66 para $25,94 \%$, quando comparado ao método convencional de propagação. Em relação ao genótipo MA701-02, os principais compostos foram a pulegona (51,56 para $68,25 \%)$ e mentona $(20,46$ para $12,69 \%)$. Nota-se um aumento da pulegona em plantas micropropagadas e uma redução da mentona nas mesmas (TAB. 5). Comparando os dois tipos de produção das plantas, nota-se que o nível de mentona foi inferior em plantas provenientes do cultivo in vitro, exceto no genótipo MA701-01, enquanto que a pulegona apresentou um nível mais elevado nesta condição.

Já em relação ao genótipo MA701-04, os constituintes majoritários em ambos os métodos foram a pulegona $(48,1$ para $55,6 \%)$ e a piperitona $(40,2$ para $31,8 \%$ ). Observa-se, assim, que houve um aumento em torno de $7 \%$ no teor de pulegona para as plantas provenientes da micropropagação (TAB. 5). A presença de uma quantidade elevada de pulegona é interessante para a produção em grande escala deste terpenóide, já que possui diversas propriedades biológicas, tais como repelente de insetos e abortivos, tanto para animais e seres humanos (TAVARES et al.., 2004). Essas variações nos teores dos constituintes do óleo essencial sugerem que a síntese desses terpenos pode está correlacionada com eventos organogênicos da planta (PHATAK, 2002 ; HEBLE, 2002) ou sob a influência de variação características ambientais.

\section{Conclusões}

1. Na micropropagação dos genótipos de M. arvensis os segmentos nodais são mais efetivos;

2. A utilização de $4,4 \mu \mathrm{M}$ de AIA promove um maior número de brotos por explante, nos genótipos MA70102 e MA701-04;

3. A concentração de $4,4 \mu \mathrm{M}$ de AIA obteve o maior número de folhas por explante no genótipo MA701-02;

4. O maior acúmulo de massa seca de parte aérea obteve-se nas concentrações de 4,4 $\mu \mathrm{M}$ AIA e 9,3 $\mu \mathrm{M}$ CIN $+8,9 \mu \mathrm{M}$ BAP $+2,2 \mu \mathrm{M}$ AIA no genótipo MA701-02;
5. O pó de coco pode ser utilizado na aclimatização das mudas dos genótipos de Mentha arvensis;

6. A micropropagação não alterou o teor de óleo essencial.

\section{Agradecimentos}

Os autores agradecem o $\mathrm{CNPq}$ e a Raros Agroindústria de Produtos Aromáticos S.A., pelo financiamento da pesquisa.

\section{Referências}

ADAMS, R. P. Identification of essential oil components by gas chromatography/mass spectrometry. Illinois: Allured Publishing Corporation. 2007. 804 p.

ALI, A. AFRASIAB, H.; SAEED, M.; IQBAL, J. An in vitro study of regeneration and micropropagation of Mentha arvensis. International Journal of Biology and Biotechnology, n.1, v. 04, p. 519-528, 2004.

ANDRADA-FRANCO, A. N.; RODRIGUEZ, A. P. M. Aclimatização da bromélia Aechmea nudicaulis em diferentes substratos. Horticultura Brasileira. v. 21, n.2, p. 158. 2003.

ASTA. Official analytical methods of the American Spice Trade Association. Englewood Cliffs: ASTA, p.8-11,1968.

CARRIJO, O. A.; LIZ, R. S. de; MAKISHIMA, N. Fibra da casca do coco verde como substrato agrícola. Horticultura Brasileira, v. 20, p. 533-535, 2002.

CHISHTI, N. et al. Clonal propagation of Mentha arvensis L. through nodal explant. Journal of Biological Scienes, v. 8, n.9, p. 1416-1419, 2006.

CORREIA, D.; CAVALCANTI JÚNIOR, A. T.; COSTA, A. M. G. Alternativas de substratos para a formação de portaenxertos de gravioleira (Annona muricata) em tubetes. Fortaleza: EMBRAPA - CNPAT , 2001.3p. (Comunicado técnico 67).

COSTA, A. S. 2006. Sustentabilidade da produção de alecrimpimenta (Lippia sidoides Cham.): micropropagação visando a conservação in vitro. Aracaju: UFS, 2006. 56p. (DissertaçãoMestrado em Agroecossistemas).

COUTO, M.; WAGNER JÚNIOR, A.; QUEZADA, A. C. Efeito de diferentes substratos durante a aclimatização de plantas micropropagadas do porta-enxerto mirabolano 29C (Prunus cerasifera EHRH.) em casa de vegetação. Revista Brasileira de Agrociência, v. 29, p. 125-128, 2003.

GRATTAPAGLIA, D.; MACHADO, M. A. Micropropagação. In:TORRES, A.C.; CALDAS, L.S.; BUSO, J.A. Cultura de Tecidos e Transformação Genética de Plantas. Brasília: Embrapa - CNPH. 1998. p.183-260.

GRISI, M. C. M. et al. Avaliação de genótipos de Mentha (Mentha ssp) nas condições de Distrito Federal, Brasil. Revista Brasileira de Plantas Medicinais, v. 08, n. 04, p. 33-39, 2006. 
GUENTHER, E. The essential oils: volume one - history, origin in plants, production, analysis. Malabar: Krieger Publishing Company, 1972. $427 \mathrm{p}$.

KUMAR, S. J. R. et al. High economic returns from companion and relay cropping of bread wheat and menthol mint in the winter-sumer season in north Indian plains. Industrial Crops and Products, v. 15, p. 103-114, 2002.

LIMA, C. S. M. et al. Influência de fitorreguladores no crescimento in vitro de partes aérea de Mentha viridis. Revista Brasileira de Biociências, v. 05, p. 669-671, 2007.

MOREIRA, M. P. et al. Efeito de substratos na aclimatização de mudas micropropagadas de abacaxizeiro cv. Pérola. Ciência e Agrotecnologia, v. 30, p. 875-879, 2006.

MURASHIGE, T; SKOOG, F. A revised medium for rapid growth and bioassays with tabacco tissue culture. Physiologia Plantarum, v. 15, p. 473-497, 1962.

OLIVEIRA, A. B.; HERNANDEZ, F. F. F.; ASSIS JÚNIOR, R. N. Pó de coco verde, uma alternativa de substrato na produção de mudas de berinjela. Revista Ciência Agronômica, v. 39, n. 1, p. 39-44, 2008.

PHATAK, S. V.; HEBLE, M. R. Organogenesis and terpenoid synthesis in Mentha arvensis. Fitoterapia, v. 73, p. 32-39, 2002.

RAJA, H. D.; AROCKIASAMY, D. I. In vitro Propagation of Mentha viridis L. from Nodal and Shoot tip Explants. Plant Tissue Culture \& Biotechnology, v. 18, n. 1, p.1-6, 2008.

ROSA, M. F. et al. Caracterização do pó da casca de coco verde usado como substrato agrícola. Fortaleza: EMBRAPA - CNPAT, 2001. 6 p. (Circular Técnica, 54).
SHASANY, A. K. et al. High regenerative nature of Mentha arvensis internodes. Indian Academy of Sciences, v. 05, p. 641-646, 1998.

SHREBSKY, E. C.; NICOLOSO, F. T.; MALDANER, J. Substrato na aclimatização de Pfaffia glomerata (Spreng) Pedersen produzida in vitro sob diferentes doses de sacarose. Ciência Rural, v. 36, p.1416-1423, 2006.

SILVA, J. V. et al. Aclimatização "ex vitro" de mudas de antúrio em diferentes substratos. Revista Ciência Agronômica, v. 38, n. 02, p. 188-191, 2007.

SILVEIRA, E. B. et al. Pó de coco como substrato para produção de mudas de tomateiro. Horticultura Brasileira, v. 20, p. 211-216, 2002.

SOUZA, R. R. et al. Avaliação de diferentes concentrações de ANA no desenvolvimento in vitro de manjericão. In: $46^{\circ}$ Congresso Brasileiro de Horticultura, suplemento CD, 2006.

TAVARES, E. S. et al. Kinetin Enhanced Linalool Production by in vitro Plantlets of Lippia alba. Journal of Essential Oil Research, v. 16, n.5, p. 405-408, 2004.

VAN DEN DOOL, H.; KRATZ, J. D. J. A generalization of retention index system including linear temperature programmed gas-liquid partition chromatography. Journal of Chromatography, v. 11, p. 463-471, 1963.

VILLA, F. et al. Micropropagação da amoreira-preta (Rubus spp.) e efeito de substratos na aclimatização de plântulas. Acta Scientifc Agronomy, v. 28, n. 1, p. 47-53, 2006. 